\title{
An Alternative Technique of Coronary Button Implantation in Patients Undergoing Modified Bentall's Procedure
}

\author{
Ujjwal Kumar Chowdhury ${ }^{1}$ Keerthi Rao ${ }^{1}$ Rajiv Narang ${ }^{2}$ Poonam Malhotra Kapoor ${ }^{3}$ \\ Minati Choudhury ${ }^{3}$ Arindam Choudhury ${ }^{3}$ Pradeep Ramakrishnan ${ }^{1}$
}

\footnotetext{
${ }^{1}$ Department of Cardiothoracic Surgery, All India Institute of Medical Sciences, New Delhi

${ }^{2}$ Department of Cardiology, All India Institute of Medical Sciences, New Delhi

${ }^{3}$ Department of Anaesthesia, All India Institute of Medical Sciences, New Delhi
}

J Card Crit Care TSS 2017;1:51-54.

\begin{abstract}
Address for correspondence Ujjwal Kumar Chowdhury, MCh, Diplomate NB, Department of Cardiothoracic and Vascular Surgery, AlIMS, New Delhi 110029, India (e-mail: ujjwalchow@rediffmail.com).
\end{abstract}

\author{
Abstract \\ Keywords \\ - alternative technique \\ - coronary button \\ implantation \\ - modified Bentall's \\ procedure
}

We present two modifications of the "button technique" and proximal aortic conduit suturing for patients undergoing modified Bentall's procedure. We interposed a glutaraldehyde-treated autologous pericardial strip between the graft and native coronary ostia during coronary ostial implantation and placed interrupted aortic annular pledgeted mattress sutures to ensure perfect hemostasis at the suture lines.

\section{Introduction}

Despite technical improvements for reconstruction of the coronary arteries by the "open button technique," graft-coronary anastomotic bleeding, proximal aortic conduit suture line bleeding, and other complications continue to occur because of the fragility and reduced tissue quality of the ascending aortic wall and connective tissue abnormality. ${ }^{1-3}$

We present two modifications of the "button technique" and proximal aortic conduit suturing on 95 patients undergoing modified Bentall's procedure in an attempt to control intraoperative bleeding and prevent later pseudoaneurysm formation complications.

\section{Technique}

Between January 1998 and March 2016, 95 consecutive patients (70 males), aged 22 to 66 years underwent modified Bentall's operation by a single surgeon (UKC) using the surgical technique described after obtaining informed consent and institutional ethics committee approval. Patients' demographic details are shown in - Table $\mathbf{1}$.
The operations were performed under moderately hypothermic cardiopulmonary bypass (CPB) through arterial cannulation (axillary artery $n=5$; femoral artery $n=90$ ) and bicaval venous cannulation through the femoral vein and direct superior vena cava. Combination of antegrade and retrograde cold blood hyperkalemic cardioplegia was used in all cases for myocardial preservation.

A Dacron composite graft with a mechanical heart valve (St. Jude Medical Inc.; St. Paul, Minnesota, United States) (conduit $25 \mathrm{~mm}, 38$ patients; $27 \mathrm{~mm}, 40 ; 29 \mathrm{~mm}, 17$ ) was used in all patients. While creating the coronary buttons, the pericoronary diseased aortic tissue was excised as much as possible leaving behind around 10 to $12 \mathrm{~mm}$ of pericoronary aortic tissue.

The composite graft was sutured to the annulus using everted, interrupted 2-0 mattress sutures over polytetrafluoroethylene pledgets (Ethicon, Cincinnati, Ohio). Each of the interrupted aortic supra-annular sutures was placed in such a fashion that successive sutures were made to pass through the previous pledget, thus ensuring perfect aortic annular hemostasis (-Fig. 1A-C). Using graft cautery, two side holes were created on the composite conduit graft measuring around
DOI https://doi.org/ 10.1055/s-0037-1606350.
Copyright $(2017$ Official Publication of License terms The Simulation Society (TSS), accredited by International Society of Cardiovascular Ultrasound (ISCU) 


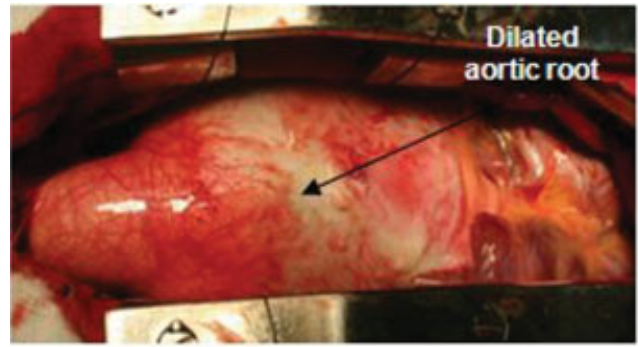

A

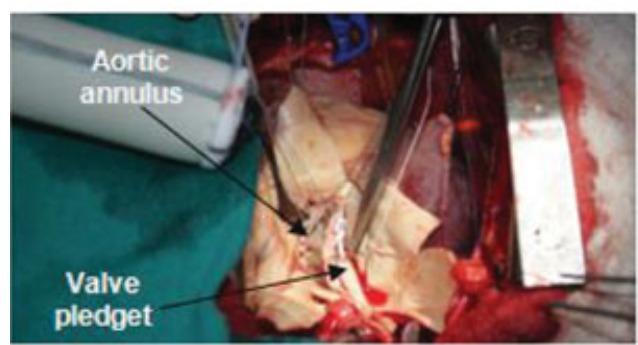

C

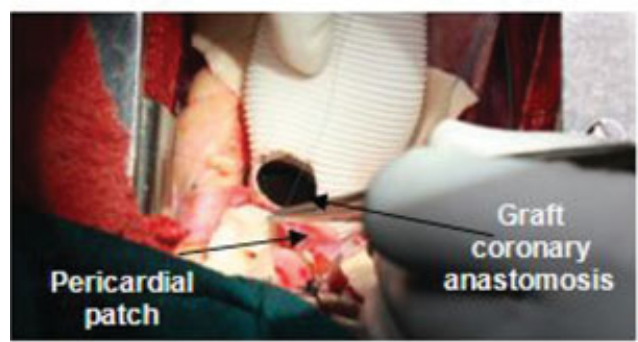

$\mathrm{E}$

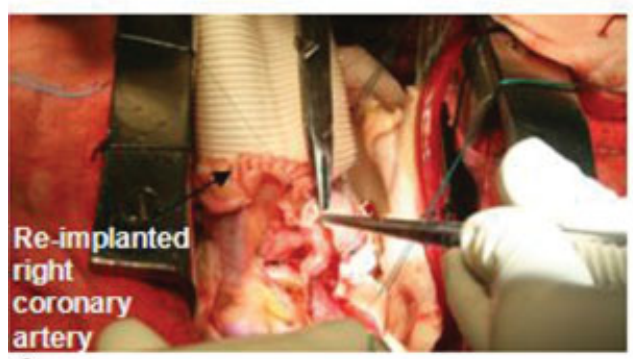

G

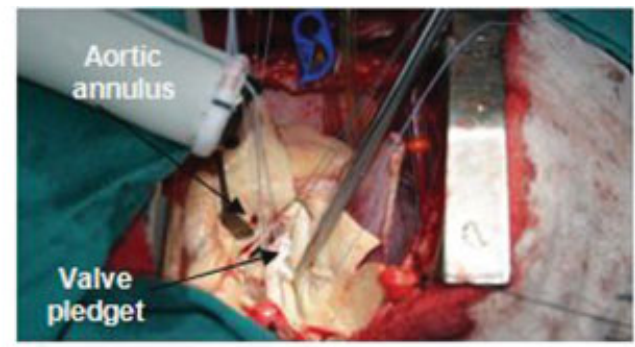

B

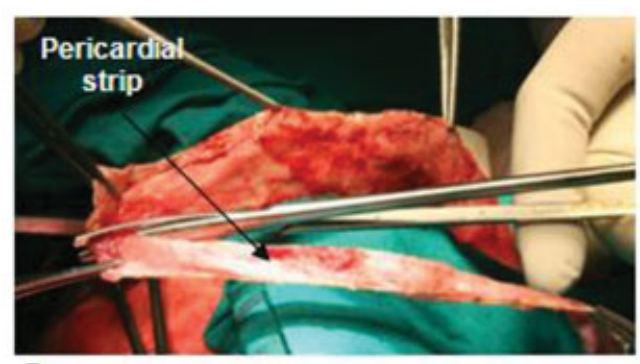

D

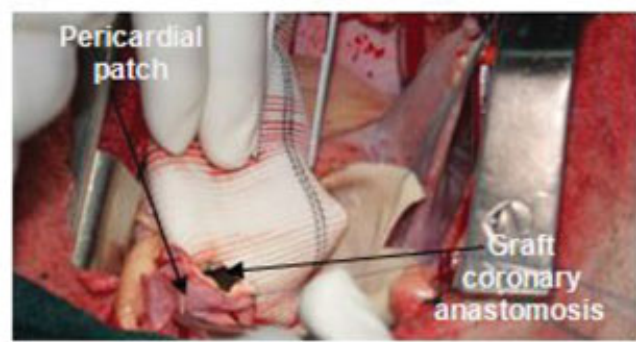

$\mathrm{F}$

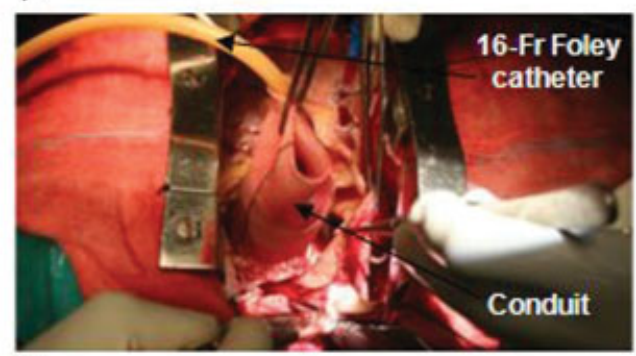

$\mathrm{H}$

Fig. 1 (A-H) Operative views of the techniques used for coronary button implantation and proximal aortic conduit suturing in a patient undergoing modified Bentall's procedure for annuloaortic ectasia. (A-C) Note the placement of interrupted pledgeted mattress sutures for proximal aortic conduit suture line. Each interrupted suture is passed through the previous pledget, ensuring perfect hemostasis. (D-G) Step-bystep demonstration of graft-coronary artery anastomosis. Note the circumferential placement of the pericardial patch between the graft and the native coronary ostium. (H) Administration of the cardioplegic solution through an inflated 16F Foley catheter inserted through the graft opening for checking hemostasis of the aortic root and coronary button anastomoses.

$10 \mathrm{~mm}$ in diameter in the proposed area of implantation of left and right coronary ostia, respectively. The left and right coronary buttons were anastomosed in an end-to-side fashion with continuous 5-0 polypropylene suture to openings on the composite graft. While suturing, a long piece, approximately 1 $\mathrm{cm}$ wide, of glutaraldehyde-treated pericardium was interposed circumferentially around each graft-coronary button anastomosis (-Fig. 1D-G).

We used topical thrombin (Tisseel, Baxter AG, Vienna, Austria) as an additional topical hemostatic agent on all patients. No aprotinin was used on any patient. The anastomoses were checked for hemostasis by pressuring the graft with cardioplegia (-Fig. 1H). The composite graft was then measured and cut to appropriate length. The distal anastomosis was performed using a running 4-0 polypropylene suture in an end-to-end fashion, on a circumferential pericardial strip, and a cardioplegia cannula was inserted through a small opening in the graft/distal ascend-

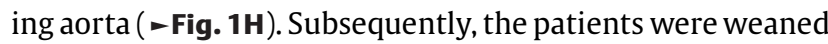
from CPB.

Mean cross-clamp time was $110.2+26.8$ minutes (range, 91-169 minutes) and mean CPB time was $142.8 \pm 25.6$ minutes (range, 124-191 minutes). All patients received warfarin and aspirin (100 mg/d) for therapeutic anticoagulation. 
Table 1 Patient profile details $(n=95)$

\begin{tabular}{|l|l|}
\hline - Annuloaortic ectasia & $68(71.6 \%)$ \\
\hline - Standard type A aortic dissection & $27(28.4 \%)$ \\
\hline - Mod to severe AR & $93(97.8)$ \\
\hline - Grade II MR & $4(4.2 \%)$ \\
\hline$\bullet \leq 40 \mathrm{EF}$ & $70(73.7 \%)$ \\
\hline - Emergency operation & $37(38.9 \%)$ \\
\hline - Renal dysfunction & $35(36.8 \%)$ \\
\hline$\bullet$ Concomitant CABG & $7(7.4 \%)$ \\
\hline
\end{tabular}

Abbreviations: $A R$, aortic regurgitation; $C A B G$, coronary artery bypass graft; EF, ejection fraction; MR, mitral regurgitation.

Three patients with acute type A dissection undergoing Bentall's procedure died in the hospital with multiorgan failure on the 10th, 14th, and 16th postoperative days. One patient with type A aortic dissection required reexploration on first postoperative day for excessive bleeding from nonanastomotic sites. The average volume of postoperative drainage was $240 \pm 70 \mathrm{~mL}$ in the first 24 hours. Seventytwo $(75.8 \%)$ patients were extubated on the first postoperative day. Mean hospitalization was $10 \pm 4$ days.

There were no late deaths. Follow-up was $100 \%$ complete (1-135 months) and yielded 912 patient-years of data with a mean follow-up time of 118.97 (standard deviation [SD] \pm 59.41 ) months. At a mean follow-up of 118.97 months, the actuarial survival was $96.8 \pm 0.02 \%$ (95\% confidence interval [CI]: 90.5-98.9). All survivors were in the New York Heart Association (NYHA) functional class I or II at their last follow-up. Postoperative studies included 6-month clinical examinations, electrocardiograms, chest radiographs, cinefluoroscopy, and echocardiography. Computerized tomographic angiography was performed when considered necessary. One patient required thoraco-abdominal aortic graft replacement with re-implantation of celiac, superior mesenteric and right renal arteries 3 years following Bentall's procedure. None of the remaining survivors have had any structural deteriorations, pseudoaneurysm formations, or reoperations.

\section{Comment}

Several technical modifications have been implemented by various investigators into the "open button technique" to minimize postoperative bleeding. These include (a) use of two separate sutures lines: an interrupted one between the most proximal part of the valve sewing ring and the aortic valve annulus and a continuous one between the more distal portion of the sewing ring and cut edge of the proximal aorta; $^{3}$ (b) harvesting of the coronary ostia with a large portion of the aortic wall, thus allowing coronary buttons to be sutured in two-layer fashion (an endo-button technique) $;^{4}$ (c) use of an additional horizontal suture line, anchoring the remnant of proximal aorta $(8-9 \mathrm{~mm}$ above the coronary ostia anastomoses) to the conduit wall (a collar technique); ${ }^{5}$ and (d) placement of a long piece of polytetrafluoroethylene felt all around the aortic annulus as well as circumferentially around each coronary button anastomoses along with fibrin glue. ${ }^{6}$

Teflon reinforcement in the aortic root has been used by others in operations for acute type A aortic dissection. However, the Teflon felt was not used as an outer supportive layer; instead, it was placed between the dissected layers of the aorta to form a neo-intima.

Despite all these modifications, postoperative bleeding from the coronary ostia anastomoses (especially located toward the aortic annulus) can still be a major concern, being difficult to control once insertion of the conduit is completed. $^{1-6}$

To improve hemostasis, pericardial strip reinforcement has been used to buttress the inflow (annular) and outflow (ascending aortic) suture lines during free-standing root replacement (Ross's operation). ${ }^{7}$ Luciani and colleagues considered the absence of pericardial strip buttressing to be an important predictor of autograft dilation.

To address these concerns, we interposed an approximately 1-cm-wide glutaraldehyde-treated autologous strip of pericardium between the graft and the native coronary ostia. The second modification was placement of interrupted pledgeted mattress sutures on the proximal aortic suture line, ensuring passage of successive sutures through the previous pledget, thus achieving perfect hemostasis. Thus, there were 10 forces driving our decision making for technical modifications to reduce/eliminate bleeding and prevent later aneurysm/pseudoaneurysm formation at the aortic root.

- The desire to perform an "open button" technique of coronary osteal implantation into the composite aortic conduit in all patients undergoing Bentall's procedure.

- The desire to minimize dissection around the proximal part of the coronary artery.

- The desire to excise as much pericoronary diseased aortic tissue from the aortic sinuses as possible.

- The desire to maintain the size of side hole of the composite graft around $10 \mathrm{~mm}$ in diameter and not according to the size of the coronary ostium.

- The desire to avoid the use of Teflon as a reenforcement material around the proximal aortic root suture line and around the coronary ostia, thereby avoiding perianastomotic excessive fibrous tissue reaction.

- The desire to interpose an approximately 1-cm-wide glutaraldehyde-treated autologous pericardial strip between the graft and native coronary ostia as a buttressing material while reimplanting the coronary arteries to the neo-aortic location on the composite aortic conduit.

- The desire to reduce the physical stress against the native wall of the fragile and diseased pericoronary aortic tissue, and as a consequence, reduce/eliminate the chances of suture cutting through causing intraoperative bleeding and/or later pseudoaneurysm formation.

- The desire to avoid the redundant native aortic wall from developing aneurysm formation at coronary osteal anastomoses in the late postoperative period. 
- The desire to strengthen the suture line around the ostium.

- The desire to obtain perfect hemostasis of the proximal aortic annular suture line by placing interrupted pledgeted mattress sutures in such a fashion that successive sutures were made to pass through the previous pledget.

We have been able to address aforementioned issues by the techniques presented here in and conclude that reinforcement of the graft-coronary anastomoses using a glutaraldehyde-treated autologous pericardial patch prevents intraoperative bleeding, pericoronary osteal fibrosis, and later pseudoaneurysm formation-a common challenge in aortic root surgeries.

Further investigations on a larger number of patients and a longer follow-up are needed to confirm the early results, especially pericoronary osteal fibrosis and late aneurysm/ pseudoaneurysm formation at the coronary anastomoses/ native coronary ostia/main trunk and/or proximal or distal aortic suture lines.

\section{References}

1 Milano AD, Pratali S, Mecozzi G, et al. Fate of coronary ostial anastomoses after the modified Bentall procedure. Ann Thorac Surg 2003;75(06):1797-1801, discussion 1802

2 Okamoto K, Casselman FP, De Geest R, Vanermen H. Giant left coronary ostial aneurysm after modified Bentall procedure in a Marfan patient. Interact Cardiovasc Thorac Surg 2008;7(06): 1164-1166

3 Copeland JG III, Rosado LJ, Snyder SL. New technique for improving hemostasis in aortic root replacement with composite graft. Ann Thorac Surg 1993;55(04):1027-1029

4 Northrup WF III, Kshettry VR. Implantation technique of aortic homograft root: emphasis on matching the host root to the graft. Ann Thorac Surg 1998;66(01):280-284

5 Nezic D, Cirkovic M, Knezevic A, Jovic M. Modified Bentall procedure-'a collar technique' to control bleeding from coronary ostia anastomoses. Interact Cardiovasc Thorac Surg 2008;7(04): 709-711

6 Sokullu O, Sanioglu S, Orhan G, et al. New use of Teflon to reduce bleeding in modified Bentall operation. Tex Heart Inst J 2008;35 (02):147-151

7 Luciani GB, Favaro A, Casali G, et al. Ross operation in the young: A ten year experience. Ann Thorac Surg 2005;80:2271-2277 